\title{
Collaboration on DIII-D Five Year Plan
}

S. Allen

April 2003

U.S. Department of Energy

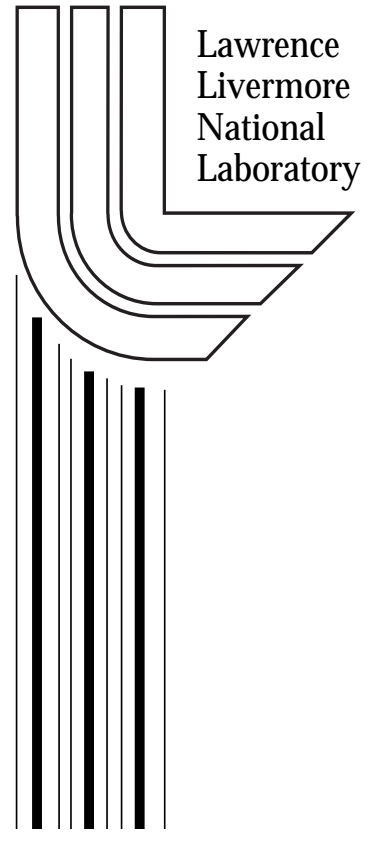




\section{DISCLAIMER}

This document was prepared as an account of work sponsored by an agency of the United States Government. Neither the United States Government nor the University of California nor any of their employees, makes any warranty, express or implied, or assumes any legal liability or responsibility for the accuracy, completeness, or usefulness of any information, apparatus, product, or process disclosed, or represents that its use would not infringe privately owned rights. Reference herein to any specific commercial product, process, or service by trade name, trademark, manufacturer, or otherwise, does not necessarily constitute or imply its endorsement, recommendation, or favoring by the United States Government or the University of California. The views and opinions of authors expressed herein do not necessarily state or reflect those of the United States Government or the University of California, and shall not be used for advertising or product endorsement purposes.

This is a preprint of a paper intended for publication in a journal or proceedings. Since changes may be made before publication, this preprint is made available with the understanding that it will not be cited or reproduced without the permission of the author. 


\section{Collaboration on DIII-D}

\section{Five Year Plan}
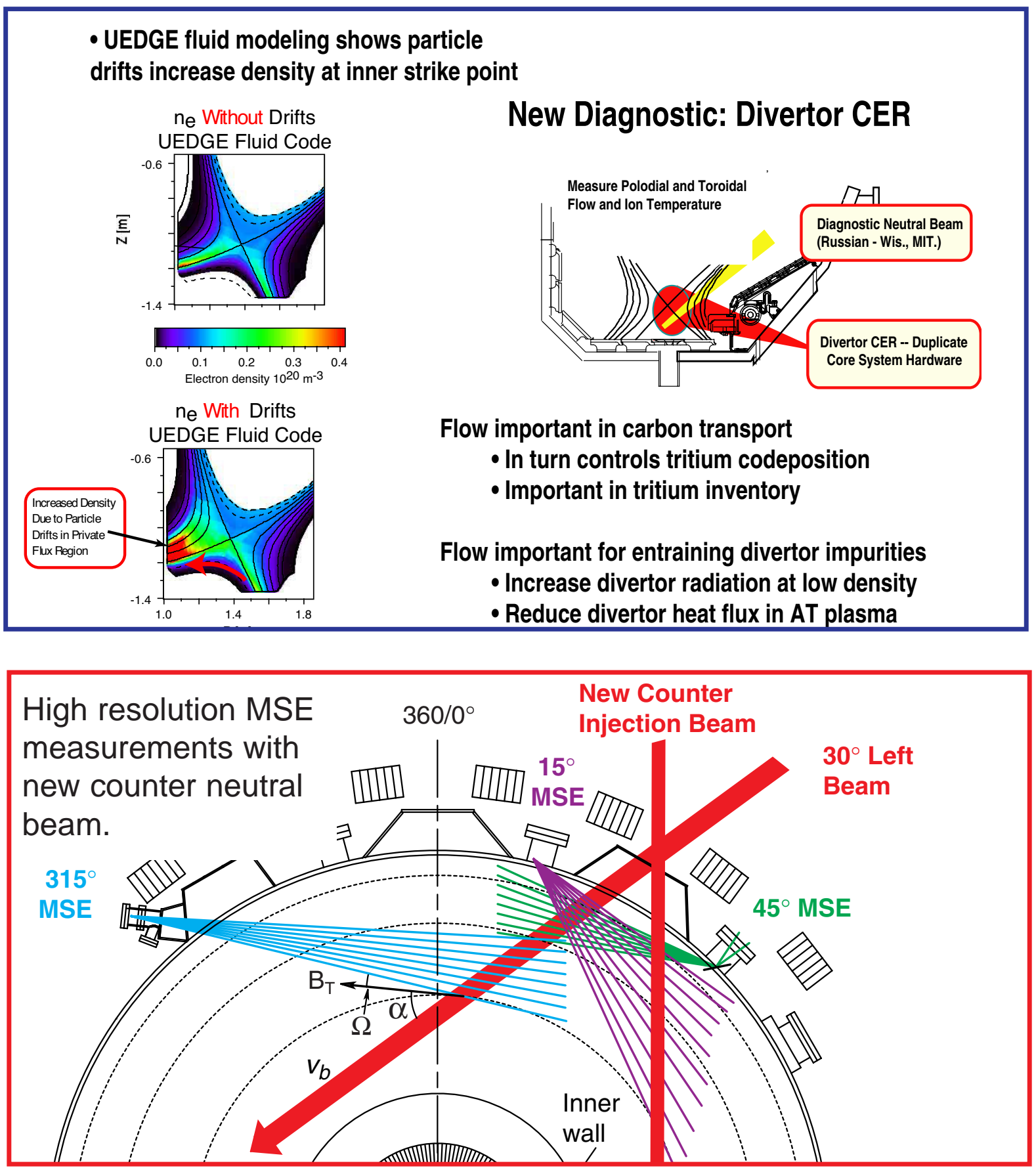


\section{EXECUTIVE SUMMARY}

This document summarizes Lawrence Livermore National Laboratory's (LLNL) plan for fusion research on the DIII-D Tokamak, located at General Atomics (GA) in San Diego, California, in the time period FY04-FY08. This document is a companion document to the DIII-D Five-Year Program Plan; which hereafter will be referred to as the "D3DPP."

The LLNL Collaboration on DIII-D is a task-driven program in which we bring to bear the full range of expertise needed to complete specific goals of plasma science research on the DIII-D facility. This document specifies our plasma performance and physics understanding goals and gives detailed plans to achieve those goals in terms of experimental leadership, code development and analysis, and diagnostic development. Our program is designed to be consistent with the long-term mission of the DIII-D program as documented in the D3DPP.

The overall DIII-D Program mission is "to establish the scientific basis for the optimization of the tokamak approach to fusion energy production." LLNL Magnetic Fusion Energy (MFE) supports this mission, and we contribute to two areas of the DIII-D program: divertor physics and advanced tokamak $(A T)$ physics. We lead or contribute to the whole cycle of research: experimental planning, diagnostic development, execution of experiments, and detailed analysis. We plan to continue this style in the next five years. DIII-D has identified three major research themes: $A T$ physics, confinement physics, and mass transport. The LLNL program is part of the $A T$ theme: measurement of the plasma current profile, and the mass transport theme: measurement and modeling of plasma flow.

In the AT area, we have focused on the measurement and modeling of the current profile in Advanced Tokamak plasmas. The current profile, and it's effect on MHD stability of the high- $\beta$ "AT" plasma are at the heart of the DIII-D program. LLNL has played a key role in the development of the Motional Stark Effect (MSE) diagnostic. Starting with a single channel, the system has grown to 40 channels with three separate systems. We have continually developed new calibration techniques, with a goal of accuracy in the magnetic field pitch angle measurements of $\sim 0.1$ degree. Measurements of the radial electric field $\mathrm{E}_{\mathrm{r}}$ have also been achieved. In the next five year period, GA plans on rotating one of the neutral beams so that it injects opposite to the sense of the plasma current (counter-injection). This enables two orthogonal MSE views of the neutral beam so that J(r) and $\mathrm{E}_{\mathrm{r}}$ can be obtained directly. In addition, the new views can be optimized so that increased spatial resolution will be obtained. Our plan is to install these new systems when the neutral beam is reoriented, and continue to provide high-resolution, "state of the art" current profile measurements for the DIII-D AT program.

In the divertor physics area, our goal is the development of a model of the scrapeoff layer (SOL) and divertor plasmas which is benchmarked with data. We have identified the need for mea- 
surements of SOL flow and ion temperature. Working with GA, we are proposing a new edge Charge Exchange Recombination (CER) diagnostic. The understanding of SOL flow is important for understanding the tritium inventory problem in ITER. In addition, using plasma flow to "entrain" impurities in the divertor region (enabling a low density radiative divertor) is the current AT divertor heat flux control scenario. We are also augmenting our edge modeling capabilities with a coupled UEDGE (fluid code) with the BOUT (edge turbulence) code. Further work, funded through LLNL theory, is planned to develop a kinetic treatment of the edge. All of these efforts contribute to the understanding of the edge pedestal in the tokamak, an important $A T$ and ITER topic.

A secondary goal is the understanding of Edge Localized Modes (ELMs), which are also important in the ITER design, as the repetitive bursts of heat flux can cause increased erosion and damage to the divertor plates. The modeling effort, particularly the kinetic treament of the pedestal region described above, is aimed at an understanding of the pedestal plasma. We plan to add fast data acquisition to several of the DIII-D edge and SOL diagnostics, e.g. the filterscopes, and imaging spectroscopic cameras, so that we can study the fast time evolution of ELMs.

\section{RESEARCH GOALS OF THE LLNL COLLABORATION}

This proposal describes the continuation of the collaborative work by Lawrence Livermore National Lab (LLNL) on the DIII-D Tokamak at General Atomics (GA). This proposal focuses on two areas of Plasma Physics research, which in turn are key parts of the overall DIII-D research program:

1)Edge physics measurements and modeling, with emphasis on the mass transport area, which is important for the ITER tritium inventory issue. In addition, the measurement and modeling of ELMs is an important topical area.

- ITER pedestal and boundary physics: Understand the physics of mass transport of carbon in an ELMing H-mode plasma to address concerns of tritium inventory in ITER and repetitive heat loads of ELMs. (DIII-D experiments and UEDGE modeling, incremental proposal for Divertor CER to measure edge plasma flow, incremental proposal for high time resolution ELM diagnostic acquisition).

-Understand fundamental transport processes in the pedestal region to more accurately predict these conditions in ITER. (BOUT fundamental turbulence code coupled with UEDGE funded with internal LLNL funds, incremental proposals for turbulence diagnostics such as gas puff imaging).

2)Measurement of the $J(r)$ and Er profile in Advanced Tokamak plasmas and the modeling of the core and edge plasma current with CORSICA. We plan to improve the MSE system when GA reori- 
ents one of the neutral beams to the counter (opposite the plasma current) direction.

- Continue development of state-of-the-art J(r) and Er measurements with MSE in ECH and ECCD experiments to measure the current profile and characterize the current drive efficiency. Design improved system that uses proposed DIII-D counter beam.

- Calculate edge current profiles with CORISCA, for comparison with new edge current measurements (GA). We also propose helping GA's development of an edge current diagnostic.

\subsection{Details of LLNL Implementation}

We bring a task oriented, team approach to this collaboration. We use a mix of disciplines on our team, and draw from the institutional strengths of LLNL. We are also part of the DIII-D research team. The highlights of our implementation plan are:

- Use of a Multiple Discipline Team. Physicists, engineers, technicians, and computational staff are part of the team. Our physics staff has experience from DIII-D, TPX, ITER, and previous LLNL experiments such as MTX, TMX-U, and MFTF-B. We use skilled engineers, technicians, and designers from the LLNL matrix system. LLNL computation staff provide important data processing capabilities. The mix of skills on the LLNL team allows us to design, construct, install, operate and process data from new diagnostics and hardware. In FY 2004 and 2005, our goal would be to restore the diagnostic engineering effort: design and build the new edge CER system, and design and build the new MSE system for the counter neutral beam installation.

- $\quad$ Strong On-site Presence of Personnel at DIII-D. This is a key component to this collabora. Several LLNL staff have leadership positions in the DIII-D team, including the RDP Leader, membership on the DIII-D Executive Committee, membership on the Research Planning Committee, and several group leaders.

- $\quad$ Use of Remote Site Operations. We are also employing enhanced communications to offsite locations (LLNL and others) using remote experimental site (distributed computing) techniques (development supported by other funding). This both enhances off-site participation, and provides additional computing resources. One example is a World-Wide-Web page to calculate EFIT reconstruction on eight computers.

- Use of Institutional Strengths of LLNL. Institutional Strengths of LLNL: We bring broader LLNL institutional strengths to the collaboration, such as the advanced computational models (UEDGE and CORSICA) developed by the MFE theory division. As needed, we will add LLNL engineering design and atomic physics expertise. We use the LLNL matrix system for engineering, computations, and technical assistance. 
- $\quad$ Participation in International Collaborations that are consistent with our research goals. We will continue to expand our emphasis on collaborative work with other international laboratories. We currently have collaborative efforts with TCV (Switzerland), JET(Helium experiments), University of Toronto, and Hampton University. Several staff are participants in the ITPA meetings for pedestal, boundary, and the confinement database. There are no additional funds provided for these collaborations.

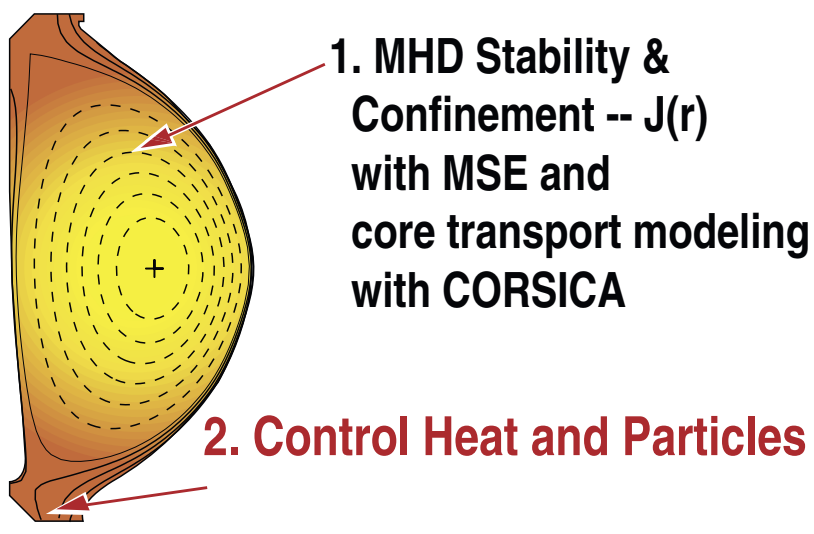

Key issues:

Tritium trapped in carbon surfaces Edge localized modes (fast MHD events) Location of recycling and impurity sources

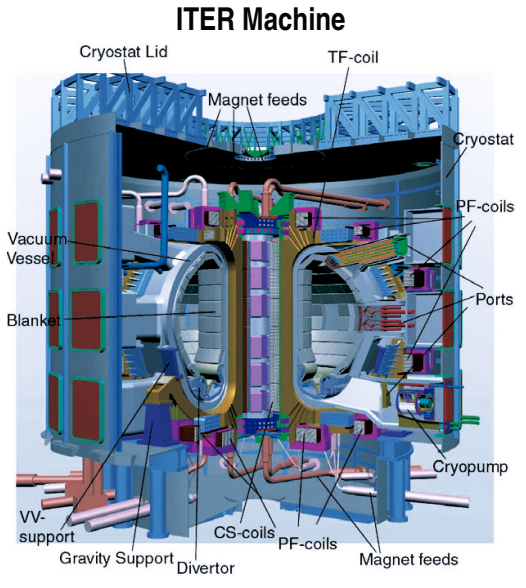
ITER Burning Plasma = Renewed Emphasis on Edge and Boundary

The LLNL Collaboration plays important roles in both the: 1) Advanced Tokamak program - MSE measurements of the plasma current profile, and the 2) Edge mass transport and boundary physics areas - we are proposing a new divertor CER measurement of plasma flow and ion temperature in the divertor and SOL. 


\section{LLNL ROLE IN THE DIII-D DIVERTOR PHYSICS PROGRAM}

This section describes the LLNL plan for advancing divertor physics research on the DIII-D tokamak. LLNL is a "full-service physics" organization in the divertor physics area. This means that our program combines:

1. Identifying new diagnostic measurements, and when necessary, building these diagnostics(Divertor CER).

2. Comparing computational models with data, and when necessary, developing the models. (UEDGE and BOUT).

3 Proposing new experiments, executing experimental run days and collecting complete datasets. Processing the raw data into physics quantities that can be related to theory and modeling.

4. Performing in-depth theoretical and computational analysis of the data to achieve detailed understanding of the physics processes involved in the experiment and the scaling of those processes to future devices

\subsection{SUMMARY OF LLNL MISSIONS AND PLANS IN DIVERTOR PHYSICS}

The mission of the LLNL program in divertor physics relates to each of these DIII-D goals:

- The LLNL divertor physics mission on DIII-D is to measure and model the critical physics processes which control SOL and divertor performance and apply this capability to the following objectives:

1. Measure and model the effect of SOL and divertor drifts, which are important in understanding the ITER tritium inventory issue and in developing an AT radiative divertor solution.

2. Develop models of edge turbulent transport, and couple these with edge fluid models. Extend these models with a kinetic treatment.

3. Measure and model ELMs so that their effect can be minimized or eliminated (ELMfree discharges such as the QH mode). ELMs present a large power burst to the divertor plate which can cause erosion at the divertor plate..

Key to this mission is to continually refine our predictive computer model of this physics by validating its results against the DIII-D divertor data. For each objective, our program uses this computer model to provide a unifying interpretation of multiple, simultaneous diagnostic measurements in terms of energy, fuel particle and impurity transport. Finally, LLNL applies this measurement and modeling expertise to divertor operation in a wide variety of toroidal devices.

The LLNL program plans to continue this mission in the next five years are summarized below. 


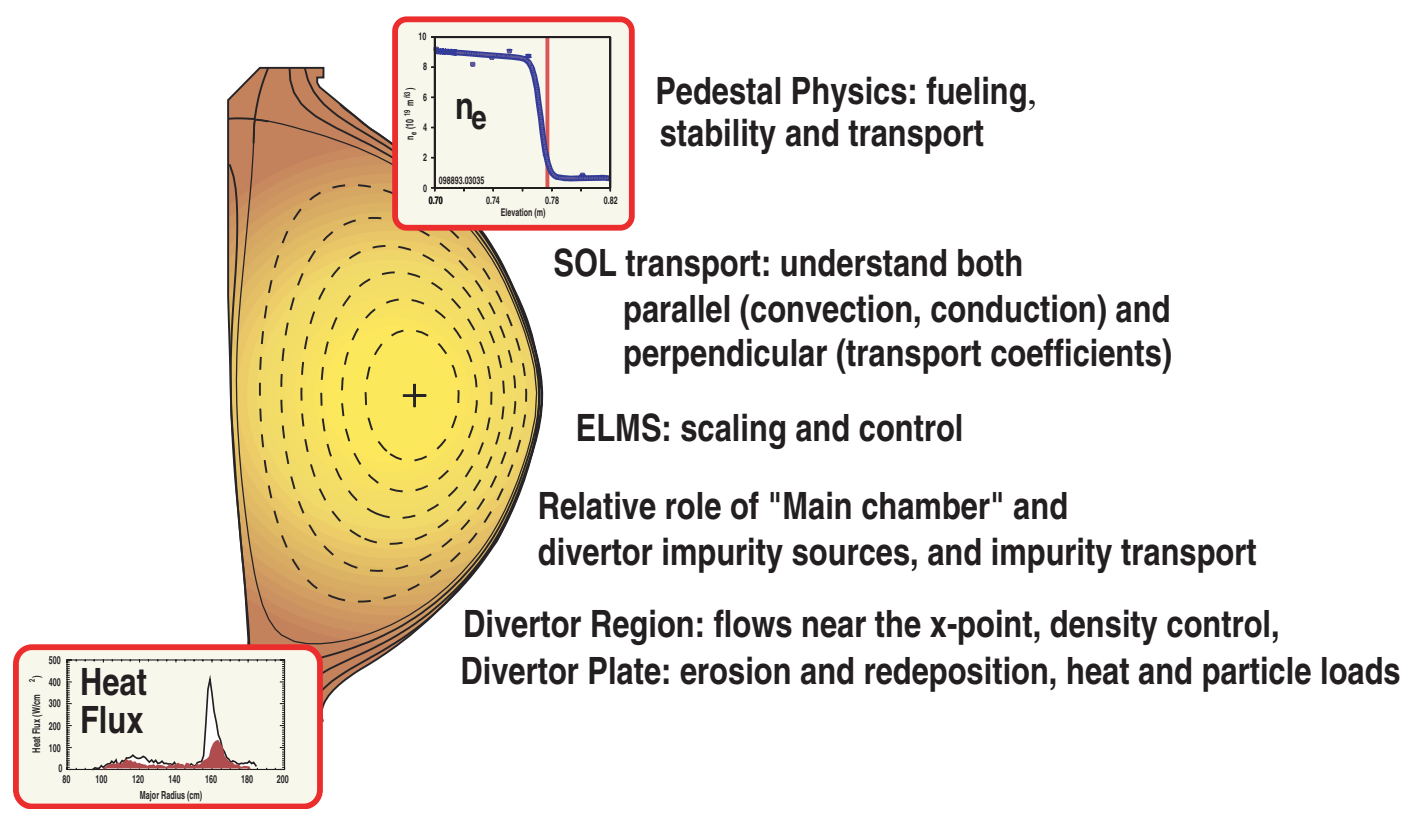

LLNL has an adtive program of physics in the core pedestal, the SOL, and into the divertor. This involves both experiments and modeling

\subsection{The DIII-D Mass Flow Initiative.}

As shown in the following table, one of the three major themes of the DIII-D Program is the massflow initiative. By "mass flow" we are trying to capture the concept that: 1) the plasma moves material (carbon in DIII-D) from one part of the vessel and 2) the plasma flow can influence where this material is redeposited. When the carbon is deposited, other elements (such as tritium) can be deposited at the same time, which can trap a large volume of tritium along with the carbon. There may be little net erosion of carbon, but a large inventory of codeposited tritium in the carbon. At the present, carbon tiles are included in the ITER design as they are very "forgiving" in the case of offnormal events such as disruptions or ELMs. The initial ITER operation is planned to be with carbon, and then later operation will either use a different material, or new information about carbon will allow it's continued use. Experiments on JET have shown that the location of the co-deposited tritium depends on the details of the divertor geometry. Initial experimentsindicated that most of the tritium was deposited in cold regions that were well separated from the plasma. More recent results, with different divertor configurations (e.g. strike point location), have shown deposition in hot, plasma facing components.

These experiments have motivated the study of the transport of carbon in the DIII-D tokamak. The flow measurements are also important for $A T$ plasma operation (discussed later), as we plan on 


\title{
Mass Transport in the Boundary, Confinement, and "AT" are three major areas of research for the DIII-D Program 5 yr plan
}

\author{
Understand the physics of mass transport in the SOL and divertor regions and \\ develop techniques to affect and control the flow of particles \\ with double null divertor capability and carbon walls
}

\begin{abstract}
Mass Transport means physically moving (carbon) in the tokamak by:
Erosion of carbon --> codeposition with tritium near walls and divertor

Plasma and impurity flow in SOL --> transporting carbon

Plasma and impurity flow in SOL --> entrain impurities in divertor (AT)

Fueling and exhaust --> density control, particularly AT
\end{abstract}

New diagnostics are required:

Erosion and reposition of carbon (quartz microbalances)

Flow measurements with CER (new)

New models are required:

Realistic x-point geometry (UEDGE-BOUT coupling)

Running existing models in a self-consistent mode

using induced flow (puffing deuterium and pumping it with cryopumps to provide a SOL flow) to entrain impurities in the divertor. This will allow increased radiation in these plasmas so that the divertor heat flux can be controlled even though the plasma density is reduced.

\subsection{The Divertor CER flow diagnostic: LLNL and GA proposal}

The experimental results on JET with carbon transport, along with UEDGE modeling of DIII-D (discussed later) indicate that plasma drifts are key ingredient in understanding mass transport in the SOL and divertor. JET measurements at one poloidal location show a large poloidal particle flow. JT-60 measurements show a complicated flow pattern, with regions of flow reversal. For this reason, we have teamed with GA in developing a measurement of the plasma flow. The plasma ion temperature in the region is also determined by the same diagnostic.

We plan to apply a technique that has been very successful in the core of the plasma to determine the plasma ion temperature and poloidal and toroidal drifts: Charge Exchange Recombination 


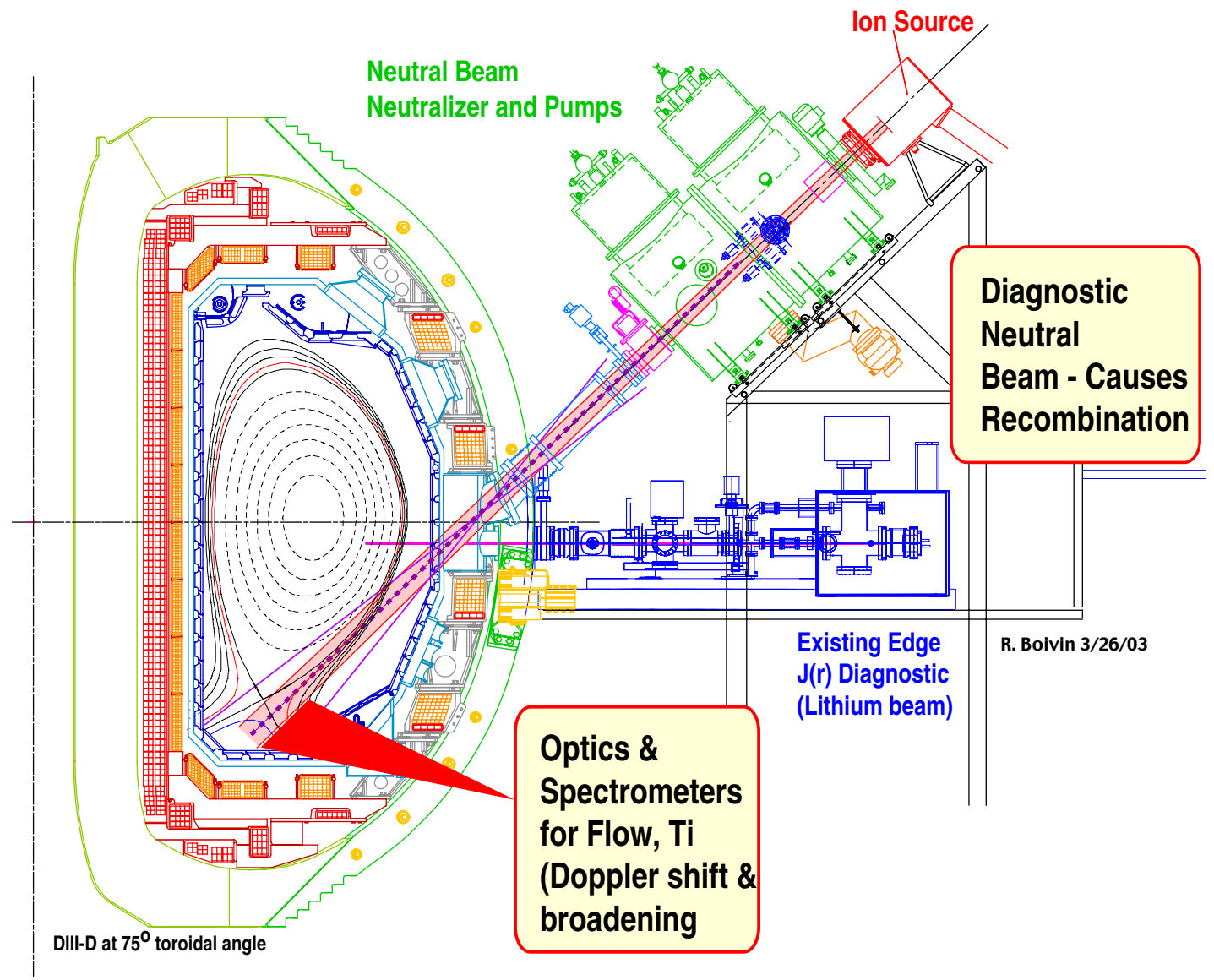

Schematic diagram of the divertor CER measurement proposed by LLNL and GA. The diagnostic neutral beam goes through the edge plasma, which enables new turbulence measurements (BES) for comparison with the BOUT code. The optics and spectrometers are similar to the core system, and measure both poloidal and toroidal components of flow. The ion temperature is also obtained.

The neutral beam has operated successfully on MST and C-MOD, and a longer duration (1.5 second) version will soon be available; quoted cost $\sim \$ 600 \mathrm{~K}$. We would use the same type of optics and detectors as the DIII-D CER core detector system. The $\$ 150 \mathrm{~K}$ for detectors is based on recent estimates by the GA staff and would include both a poloidal and toroidal array. A LLNL diagnostic engineer would design and construct the Divertor CER system, with designer help from GA in FY04-FY05. 
spectroscopy. A neutral beam is injected into the plasma, which causes a local recombination of fully stripped carbon, which then radiates. The doppler width and doppler shift of these emissions are then used to determine the ion temperature and plasma rotation in the plasma core.

A schematic of the proposed system is shown in the following figure.

\subsection{The suite of mass flow diagnostics.}

The divertor CER is a key measurement in the mass flow initiative, but several other diagnostics are critical, as shown in the following figure. We plan to diagnose the four processes:

1. Carbon influx from the walls -- quartz microbalances and CCD cameras

2. SOL flow -- divertor CER

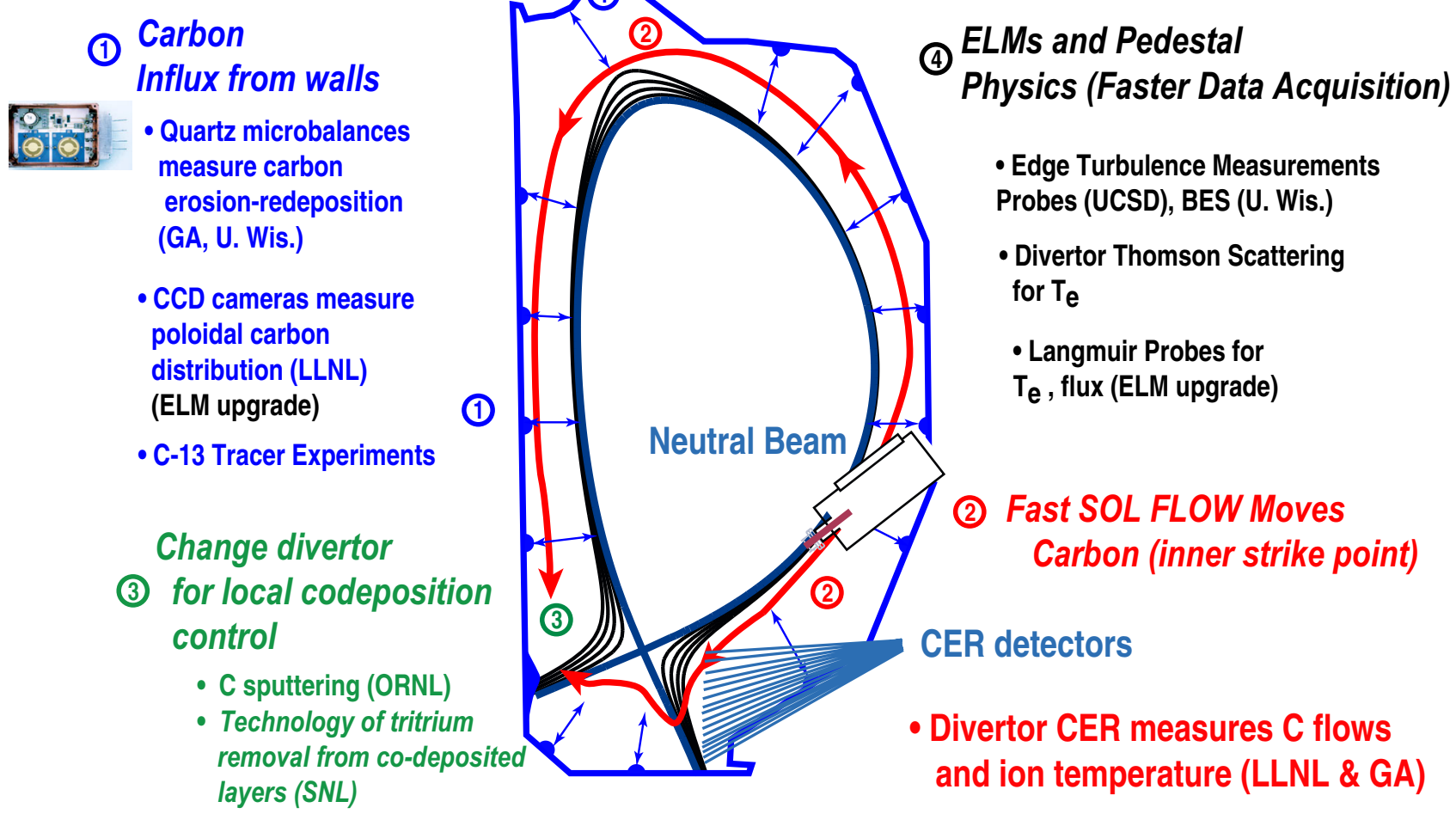

The suite of diagnostics proposed for the mass flow initiative. Measurments and modeling of 1) the carbon influx from the walls, 2) SOL flow, 3) Local divertor processes, and ELMS and pedestal physics are important 
3. Divertor geometry -- carbon sputtering(spectroscopy), and tritium removal

4. ELMs and pedestal physics -- increased time resolution measurements.

\subsection{Other LLNL Edge and Boundary Goals}

1) 2003-2008. Divertor Experiments and analysis: Our physics staff will plan and carry out divertor experiments focused on understanding the mass flow of carbon in the all-carbon DIII-D tokamak. These data are critical for understanding the tritium inventory problems in ITER.

2) 2003-2008. Divertor Diagnostic Operations: Operate and maintain several divertor diagnostics, IRTV's, 3 tangential TV systems, and the DIII-D Bolometer system.

3) 2003-2008. UEDGE code modeling with drifts: We will continue to compare DIII-D experimental data with the UEDGE code.

4) 2003-2008. Operate UV Camera for upper and lower divertors, with Hampton University. Compare CIV in open and closed divertors.

5) 2003-2008. DIII-D Program Management: LLNL personnel serve as Thrust leaders, and a LLNL person is the DIII-D Divertor Science Group Leader

\subsection{LLNL Activities in Edge and Boundary Modeling}

The LLNL program is a balance between measurements and modeling. We have extensive capabilities in both fluid modeling (UEDGE) and turbulence modeling .Under LLNL funding, we are currently coupling the UEDGE and BOUT code. LLNL also has plans continue to extend the (collisional) BOUT code to a more kinetic treatment of transport, which is more appropriate for the edge of

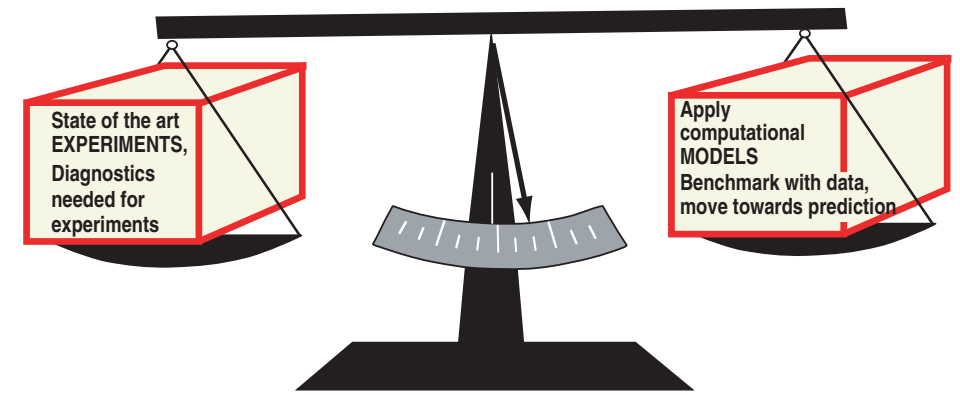

Edge and Divertor Experiments:

Development of key measurements:

$\mathrm{T}_{\mathrm{e}}, \mathrm{n}_{\mathrm{e}}$, heat flux, 2-D impurities

New fast ELM diagnostics

Proposed: Measurement of $\mathrm{T}_{i}$, flow with CER

Measurement of SOL transport - probes, gas

puff imaging

Divertor geometry modifications for $\mathrm{n}_{\mathrm{e}}$ control in high- DN
Boundary Modeling:

UEDGE fluid code - benchmarked BOUT turbulence - $x$-point geometry EIRENE (neutrals) - collaboration DIVIMP (impurities) - Univ. Toronto Core Modeling: CALTRANS - GA \& LLNL transport Core - Edge Coupled models UEDGE-BOUT edge modeling 
- UEDGE

- 2-D fluid transport model

- Widely used for

- Experimental analysis

- Edge modeling

- BOUT

- 3-D edge turbulence model

- Braginskii fluid equations

- Realistic magnetic geometry

- Benchmarked against Expt.

- DIII-D L-mode

- C-Mod QC-mode

- Density limit

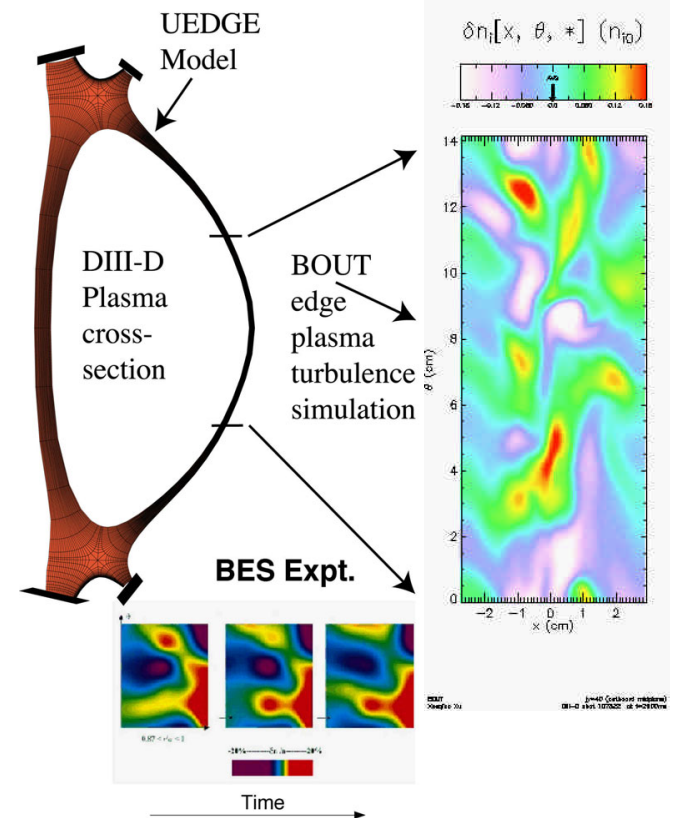

DIII-D. The two codes are described in the figure above, and the plans for the coupled code and included in the table. Much of the work is funded on internal LLNL funds, but there is also a proposal to the ISOFS for funding the kinetic work.

\section{BOUT/UEDGE Coupling}

- UEDGE - a 2-D fluid model of the plasma edge

- Accurate treatment of

- Neutrals, (plasma recycling, neutral penetration, ionization source, etc)

- Impurities (sputtering, impurity radiation, multiple charge states, ...)

- Transport equation for toroidally averaged profiles of $n, T, \phi, \ldots$

- Empirical transport coefficients to model effect of turbulence

- Benchmarked against experiment

- BOUT - a 3-D fluid model of plasma edge turbulence

- Fluid plasma edge turbulence in realistic magnetic geometry

- Needs better treatment of neutral sources, impurity radiation, ...

- Run BOUT with fixed toroidally-averaged profiles to benchmark against Expt.

$\Rightarrow$ Couple BOUT with UEDGE to model effect turbulence on transport

- Self-consistent neutral sources, impurity radiation, etc.

- Self-consistent treatment of turbulent transport and profile evolution (this effort is proceeding under LLNL internal funding) 
Elements of the Boundary computational plan. These include the coupled UEDGE and BOUT codes, followed by a development of a kinetic edge code. The plan is set up so that intermediate results can be obtained, both to provide checkpoints along the way, and also to motivate other funding sources such as ISOFS. This plan will be carried out by LLNL theory, but the DIII-D collaboration provides personnel to interface the code results with experimental data. Physicists on the collaboration at currently doing this important benchmarking work for UEDGE, BOUT, and the CORSICA core transport code.

\section{Boundary Computational Plan}

- Couple BOUT and UEDGE (in progress on LLNL funding)

- Density limits

- Structure of H-mode pedestal

- Quantitative at high collisionality

- Qualitative at low collisionality

- Develop Edge Kinetic Code (proposed for LLNL funding)

- Two stages:

- 2-spatial, 2 velocity dimensions

- 2-spatial, 3 velocity dimensions

$\Rightarrow$ Structure of $\mathrm{H}$-mode pedestal for ITER-relevant parameters

$\Rightarrow$ Accurate projections of ITER fusion gain
- In parallel (also on LLNL funding)

- Physics-based multi-scale models of plasma wall interactions

- Define interface for coupling MD with plasma edge simulations

- Plan to ISOFS funding

Oct. 2005

- Expand team (to include broader MFE community participation)

- Expand funding

- Code validation

- Physics exploration

- More comprehensive model 


\section{LLNL ROLE IN THE DIII-D AT PROGRAM}

The overall goal of the DIII-D AT program is to develop a quasi-steady state plasma with high confinement $(\mathrm{H}=4)$ and high beta $\left(\beta_{\mathrm{N}}=6\right)$ with near $100 \%$ noninductive current drive $(\mathrm{H}$ is defined here as the energy confinement time relative to the ITER-89 p-scaling). In recent experiments on DIII-D, the parameters $\mathrm{H}=4$ and $\beta_{\mathrm{N}}=6$ have been obtained, but for time periods less than an energy confinement time $(\sim 0.5 \mathrm{~s})$. The best long-pulse discharges have $\mathrm{H}=2.4$ and $\beta_{\mathrm{N}}=2.8$ for $\tau>8 \tau_{\mathrm{E}}$. The key to improving tokamak performance from the existing state towards the long-term goal of $\mathrm{H}=4, \beta_{\mathrm{N}}=6$ is controlling and optimizing plasma profiles of parameters such as the current density (or $q$ ), pressure, and $\mathrm{E} \times \mathrm{B}$ shearing rate.

Recent experiments on DIII-D have demonstrated the potential improvement in performance through profile modification. ECCD has been used to stabilize Neoclassical Tearing Modes, and to directly modify the current profile.

To make progress in the AT area, our program requires four elements:

1. Scientific understanding of the effects of heating, current drive and particle fueling on profiles and their affect on stability and confinement.

2. Accurate profile measurements to guide experiments, with current density of particular interest.

3. Transport analysis and comprehensive modeling to develop and extend duration of AT scenarios.

4. Feedback control of profiles to ultimately sustain high performance regimes.

In the area of profile measurements, we have developed a 35-channel MSE diagnostic over the past six years. This instrument, in combination with the EFIT equilibrium code, provides accurate reconstruction of both the $q$-profile and the radial electric field $\left(\mathrm{E}_{\mathrm{r}}\right)$ profile. These measurements have been absolutely critical to the development and understanding of AT regimes in recent years. To support this effort, we are actively involved with enhancements to the analysis features of EFIT and its execution efficiency for use during operations.

The development of the CORSICA modeling code brings to the AT program a tool for comprehensive modeling of time-dependent processes and affords the capability for predictive and core-edge coupled calculations. The evolution of current and transport profiles predicted by CORSICA have recently been benchmarked against real experimental data with reasonably good results. This code is a valuable tool for developing future AT scenarios. Finally, we will use our measurement capability in combination with scenario development from the CORSICA code to do feedback control of profiles ( $q$, pressure, $\mathrm{E} \times \mathrm{B}$ shear) with the aim to improve pulse length and overall performance of AT modes. Feedback control requires profile control tools such as rf and neutral beam heating and current drive. The DIII-D research plan calls for increasing the ECH power to $6 \mathrm{MW}$; this increased power should enable further progress in the realization of active profile control. 


\subsection{MOTIONAL STARK EFFECT DIAGNOSTIC}

One significant recent development with the MSE diagnostic is the realization that the plasma radial electric field $\left(\mathrm{E}_{\mathrm{r}}\right)$ can affect the MSE measurement. In past work, it was generally true that $\mathrm{E}_{\mathrm{r}}$ was small compared to the large motional $\mathrm{E}=v_{\mathrm{b}} \times \mathrm{B}$ electric field experienced by the high-energy neutral beam atoms. However, recent NCS and VH-mode plasmas have $\mathrm{E}_{\mathrm{r}}$ fields up to $200 \mathrm{kV} / \mathrm{m}$ which significantly affects the MSE measurement.

It can be shown that if the MSE diagnostic is modified to measure the pitch angle at a given radial location with two separate viewing geometries, then the $E_{r}$ field can be determined directly using MSE. Since shear the $\mathrm{E}_{\mathrm{r}} \times \mathrm{B}$ flow is the leading model for explaining the improved transport observed in NCS and other high-performance discharges, a direct measurement of $\mathrm{E}_{\mathrm{r}}$ would be of great importance.

During the 1997 vent on DIII-D, the MSE system was upgraded from 16 to 35 channels with the addition of a completely new radial view. This new radial view provides the second view necessary to resolve the $\mathrm{E}_{\mathrm{r}}$ field. An overview of the current MSE viewing sightlines is shown below.

The DIII-D program plans to modify one of the neutral beam injectors so that it injects opposite to the normal direction of the plasma current (counter-injection). With the present system, all of the

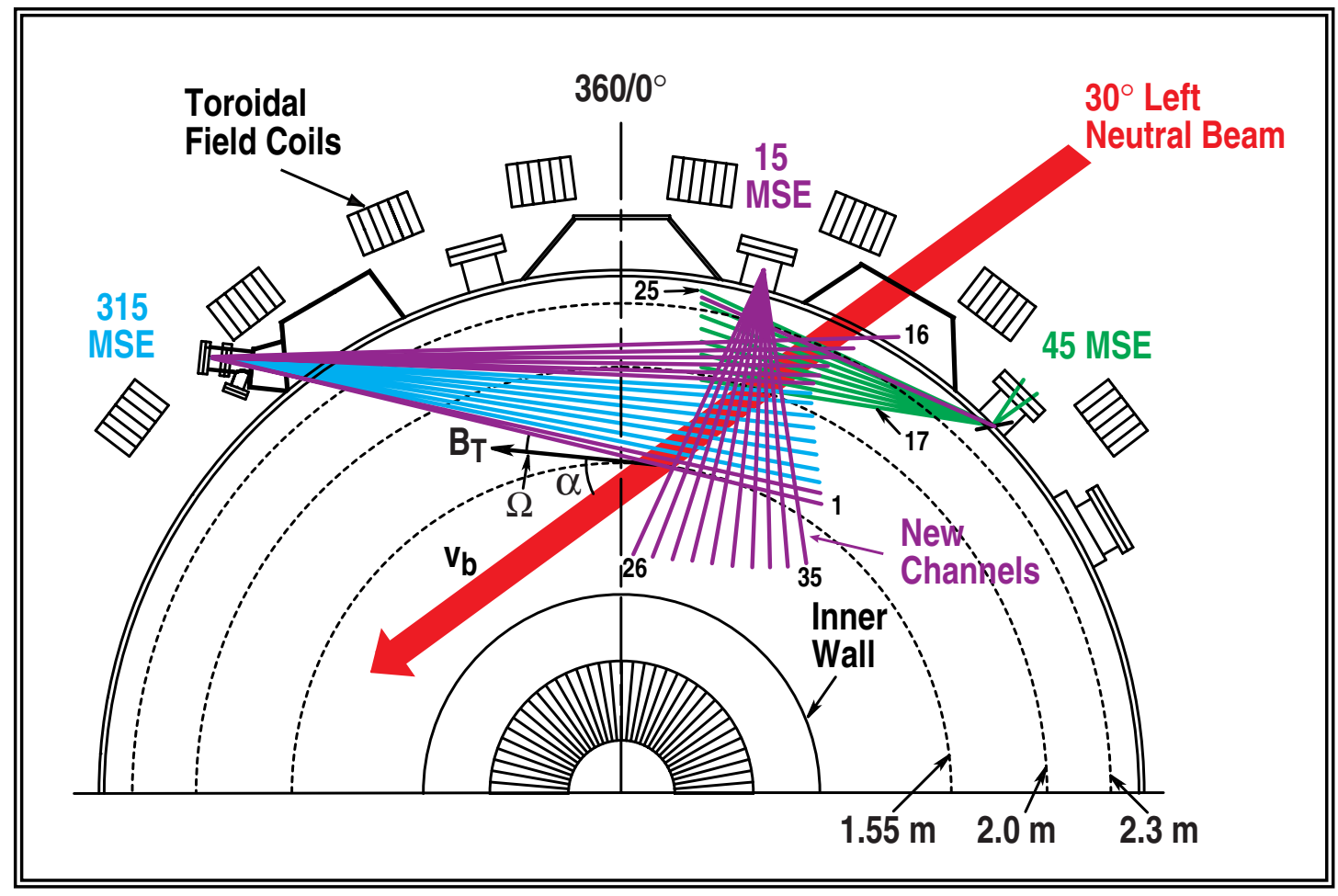

Viewing geometry of the upgraded MSE system. The view from the port at 15 deg was added during the last vent to help resolve the $E_{r}$ profile 
sources are either co- or counter- inection (relative to the plasma current). When this modification is made, it allows simultaneous MSE measurements and the $\mathrm{E}_{\mathrm{r}}$ profiles can be obtained directly. In addition, we will be able to modify the system to achieve better spatial resoltuion.

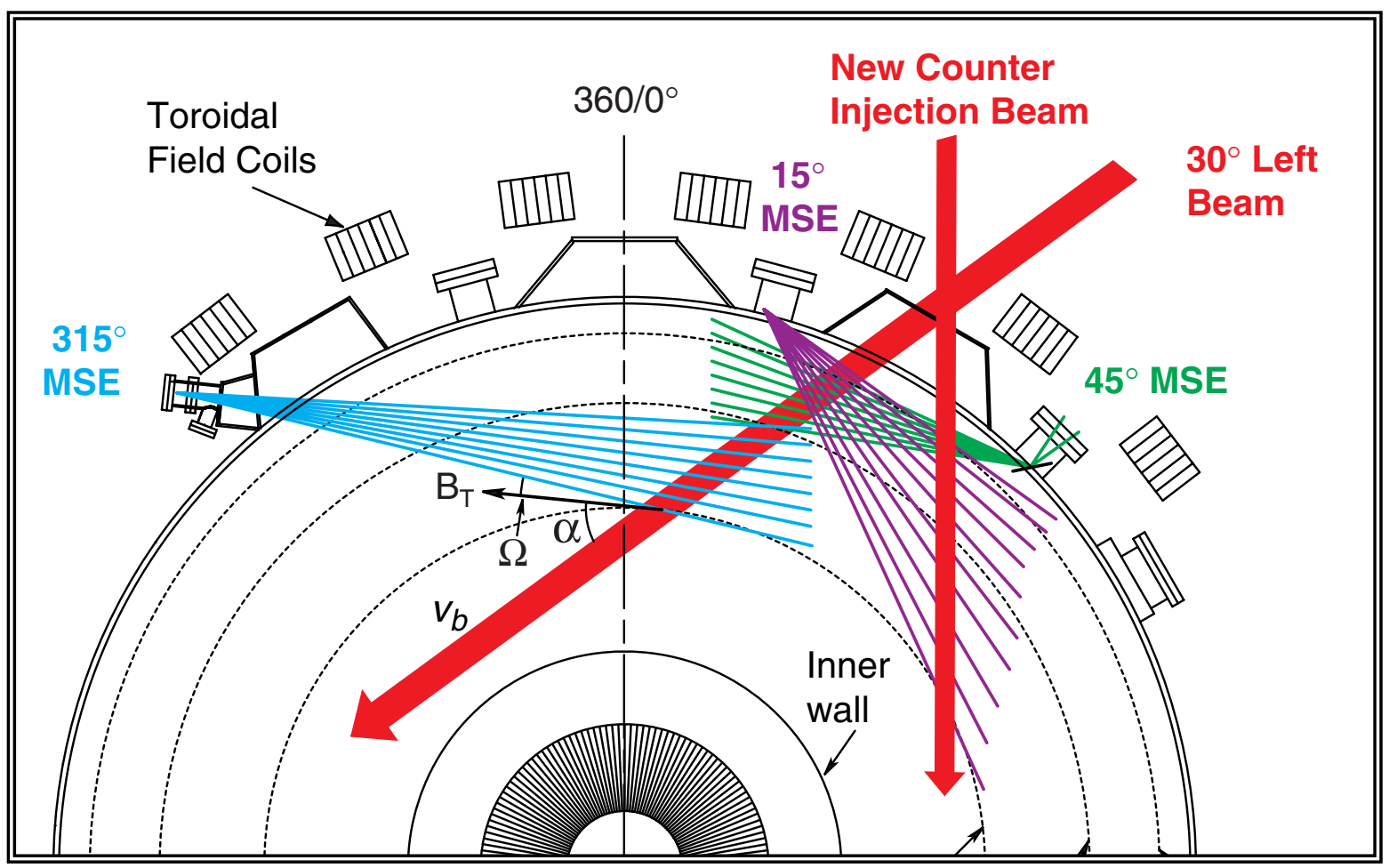

With the addition of a counter beam, optimum radial resolution is obtained for the measurement of $q(r)$ and $E r$.

Feedback control of the $q(\mathrm{r})$ and $\mathrm{E}_{\mathrm{r}}$ profiles for optimizing stability and transport is necessary to sustain a high performance AT plasma. The MSE signals can be used for feedback control of both $q(\mathrm{r})$ and $\mathrm{E}_{\mathrm{r}}$. At this time, we have fiber optic links for $16 \mathrm{MSE}$ channel signals for feedback to the plasma control system (PCS). Using these signals, the real-time calculation of $q(0)$ has been demonstrated using an algorithm developed by LLNL. The real-time running of EFIT (without MSE) has also been demonstrated.

CORSICA is a comprehensive simulation code developed at LLNL using internal funding to model toroidal plasma confinement systems. It couples calculation of 2-D equilibria with 1-D core transport models to provide time-dependent modeling uniquely suited to studying the evolution of AT modes of tokamak operation. Equilibrium solutions have been benchmarked against fits to experimental equilibria produced by the EFIT code and compared with $q$-profile evolution in DIII-D (neoclassical resistive current evolution) using MSE-based data analysi. Several different transport models are currently available and CORSICA's modular structure allows user customization and addition of transport models. CORSICA can be run in a predictive mode using transport models or constrained by experimentally measured profiles of density, temperature and/or Z-effective as desired. 


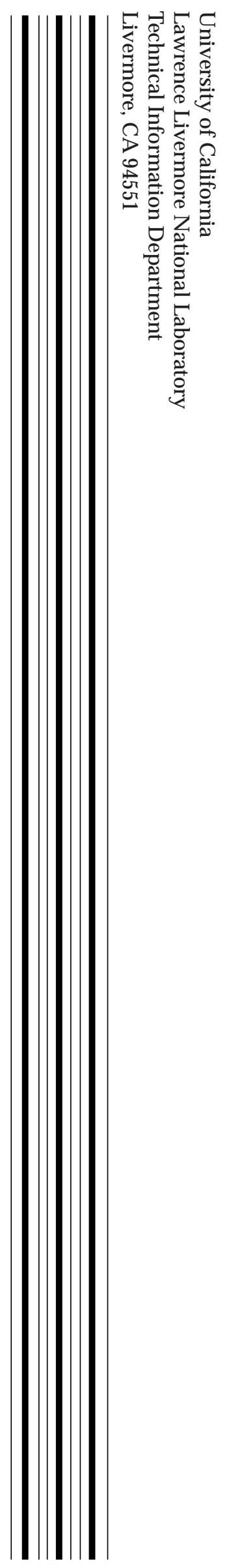

